\title{
Performance Analysis of Texture Image RETRieval For Curvelet, CONTOURlet TRANSFORM AND LOCAL TERNARY PATTERN USING MRI BRAIN TUMOR IMAGE
}

\author{
A. Anbarasa Pandian and R. Balasubramanian \\ Department of Computer Science and Engineering, \\ Manonmaniam Sundaranar University, Tirunelveli, India
}

\begin{abstract}
Texture represents spatial or statistical repetition in pixel intensity and orientation. Brain tumor is an abnormal cell or tissue forms within a brain. In this paper, a model based on texture feature is useful to detect the MRI brain tumor images. There are two parts, namely; feature extraction process and classification. First, the texture features are extracted using techniques like Curvelet transform, Contourlet transform and Local ternary pattern (LTP). Second, the supervised learning algorithm like Deep neural network (DNN) is used to classify the brain tumor images. The Experiment is performed on a collection of 1000 brain tumor images with different orientations. Experimental results reveal that contourlet transform technique provides better than curvelet transform and Local ternary pattern.
\end{abstract}

\section{KEYWORDS}

CBIR, Texture, Curvelet, Contourlet, Local ternary pattern and Deep neural network.

\section{INTRODUCTION}

In the early years, advances in modern computer and telecommunication technologies have led to huge archives of multimedia data in diverse application area such as medicine, remote sensing, entertainment, education and on-line information services. Due to increasing the amount of alphanumeric data which led to the development of database management systems (DBMS). In DBMS, organizing the alphanumeric data into interrelated collections so that information retrieval and storage could be convenient and effective. This technology is not well suited to the management of multimedia information. The available multimedia information can be effective, efficient methods for storage, browsing, indexing and retrieval must be developed.

Since 1970, image retrieval is a very active research area within two research communitiesdatabase management and computer vision. When the size of the image database becomes very huge then two problems are manual annotation ineffective. The first problem is the amount of labor involved in image annonation. The other problem is capturing the rich content of images using a few keywords, a difficulty is compounded by the subjectivity of human perception. Content based image retrieval (CBIR) is to overcome these difficulties for large collection of images. In CBIR, features are automatically extracted and indexed by summarizing their visual contents like texture, color and shape [1]. The MRI uses radio waves for originally to brain images like bleeding, aneurysms, tumors and damages. The MRI is an accurate procedure for hundreds of images in slices per single patient. The major tissues in MRI brain images are ray 
matter, white matter and cerebro-spinal fluid. The T1, T2 and PD are the images produced to specific tissue characteristics of the image. The three types of image orientation in brain images are coronal, sagittal and axial. The medical image is used in modern techniques like Digital radiography (X-ray), ultrasound, microscopic imaging, computed tomography (CT), magnetic resonance imaging (MRI), single photon emission computer tomography (SPECT) and positron emission tomography (PET) [2].

\section{RELATED WORK}

Wells et al, [3] a new fully automatic adaptive segmentation method is used for intensifying correcting and segmenting MR images. The expectation-maximization (EM) algorithm that allow is more accurate segmentation of tissue in magnetic resonance imaging (MRI) data. Intra and Interscan intensity inhomogeneities are done on adaptive segmentation. It increases the robustness and level of automation for the segmentation of MR images.

Unay et al, [4] a region of interest retrieval system based on local structure using MR brain images. To overcome the problem of intensity, invariant structure features are LBP and KLT are used. This method is fast and robust, has high accuracy, and does not require registration, intensity normalization, or bias field correction.

Shen et al, [5] to integrate object appearance and region information on parametric deformable model is active volume model and multiple surface active volume model method is used. AVM method can perform segmentation efficiently and reliably on CT, MRI and ultrasound images with flexible initialization and rapid convergence. The main advantage of AVM and MSAVM are extracting boundaries of organs, such as lung, heart and brain for medical images.

Selvathi et al, [6] has developed the Texture features like gray level co-occurrence and statistics are extracted using MRI brain data. The Classification is based on advanced kernel based techniques such as Support Vector Machine (SVM) and the Relevance Vector Machine (RVM) is used for normal and abnormal are deployed.

Caiet al, [7] an efficient 3D content based neurological image retrieval for FDG PET images. The texture features are extracted CMRGlc (cerebral metabolic rate of glucose consumption) with volumetric co-occurrence matrix. The advantages are data analysis, robust and efficient retrieval of relevant clinical cases.

$\mathrm{Li}$, Jin et al, [8] in order to overcome the problem of semantic approach medical images is used. The low level feature like texture and color are extracted based on region of interest. The semantic correlation method is used to retrieve CT brain images.

Li, Weijuan et al, [9] has proposed a meticulous classification is done for MR-brain images using support vector machine (SVM). The texture and shape feature are extracted using statistical association rule miner algorithm to compute weight coefficients of each feature.

\section{FEATURE ExTRACTION}

Feature extraction is a dimensionality reduction that starts with the initial set of measured data for human interpretations. Feature extraction is used to reduce the huge set of data from the resources. 


\subsection{Texture}

Texture represents spatial or statistical repetition in pixel intensity and orientation. Texture analysis is an important issue used in a variety of image and video applications including image segmentation, image retrieval, object recognition, contour detection, scene classification and video indexing. Texture feature are visual pattern consist of contrast, uniformity, coarseness, and density. In CBIR, The statistical texture features are used in the edge histogram descriptor (EHD), gray-level co-occurrence matrix (GLCM), MPEG -7 texture descriptor and wavelet moments. Multiresolution representations of the transforms are consistent with the human perception of images. Multiscale transform-based methods include the Gabor transform, the wavelet transform, the ridgelet transform and the contourlet transform. In medical image analysis, the main objective of the texture is used to classify the brain images into gray and white matter of the magnetic resonance (MR) image or to detect cysts of the kidney in X-ray and computed tomography (CT) images [10].

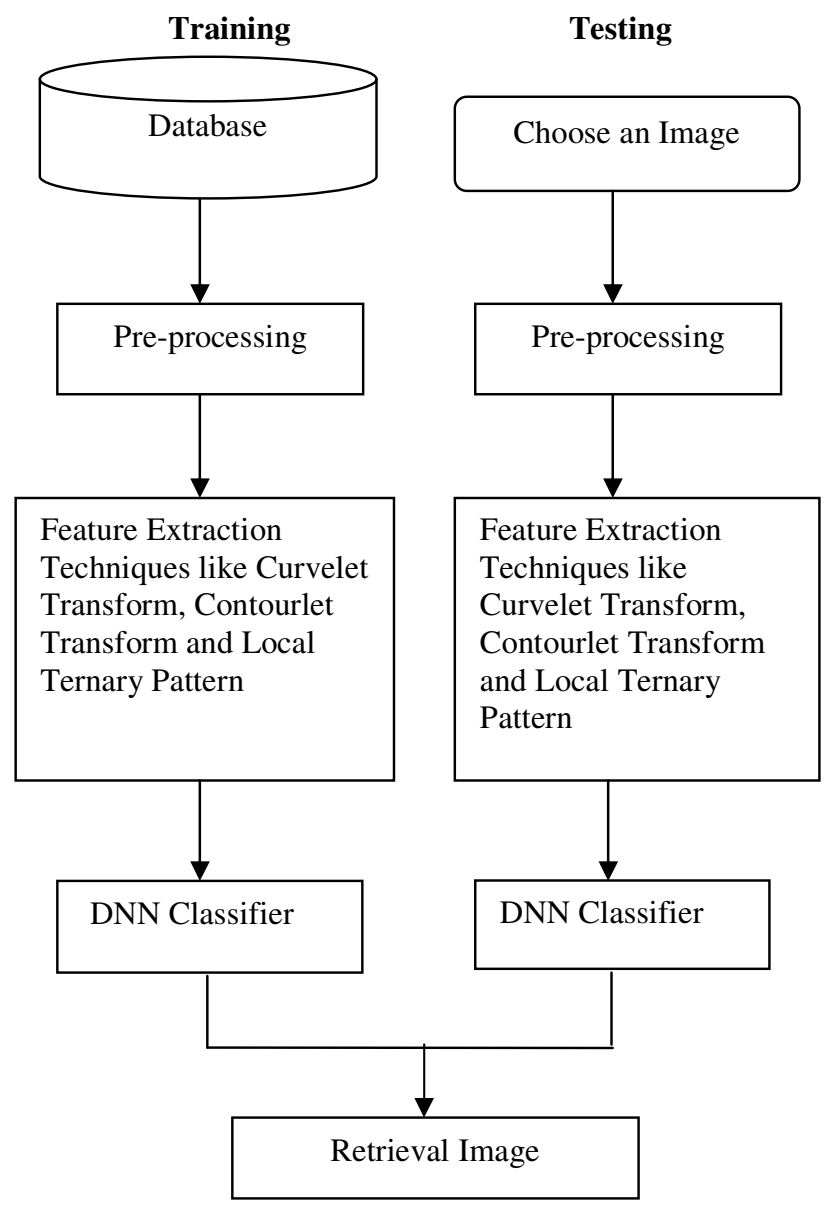

Fig. 1 Flowchart of Texture feature extraction

\subsection{Pre-processing}

The Preprocessing step is used to enhance the visual appearance of an image using contrast enhancement techniques. The Contrast enhancement is used to perform adjustments on the darkness or lightness of the image. 


\subsection{Feature extraction techniques}

The texture feature is extracted using feature extraction techniques like curvelet transform, contourlet transform and local ternary pattern

\subsection{Curvelet Transform}

Curvelet Transform (CT) represents a curve like features the use of texture and spatial locality information for motorcycle directional transforms. The digital image processing is important to handle brain visual cortex of images as spatial locality, scale and orientation. CT is used in the 3$\mathrm{D}$ biological data analysis, seismic data analysis, fluid mechanics analysis, video processing and partial differential equation analysis. CT is locally implemented ridgelets and is closely related to ridgelets. CT can represent even curve like features sparsely, whereas ridgelet sparsity is on the straight line like features. Curvelet-based feature extraction is defined as the extraction of characteristic and discriminating curvelet coefficient features from the data [11], [12].

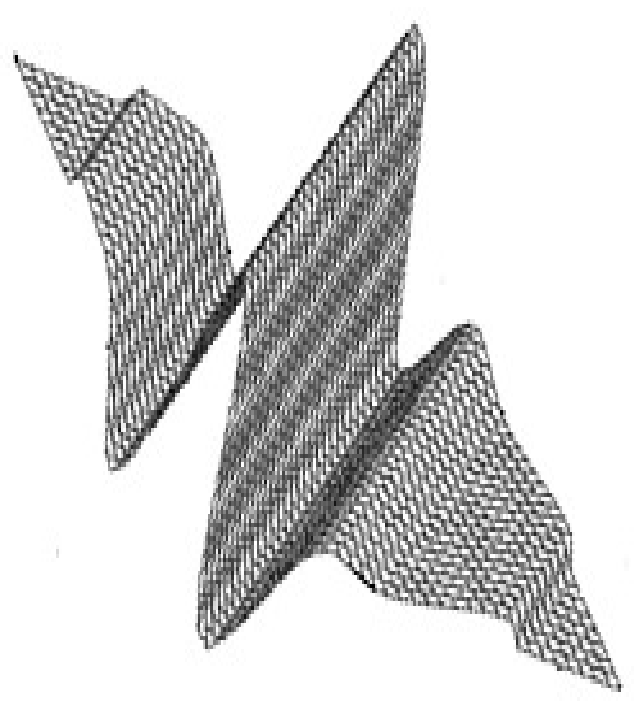

Fig. 2a Ridgelet waveform

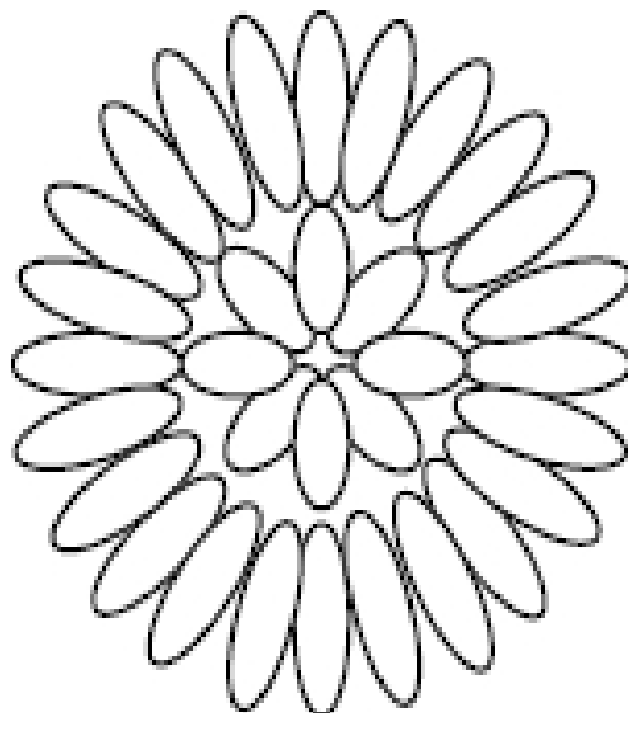

Fig. 2b Curvelets with different scales

\section{Curvelet Transform algorithm}

1. To calculate the Fourier transform of $\mathrm{f}$ by means of a $2 \mathrm{D}$ FFT

$$
f\left[n_{1}, n_{2}\right],-n / 2<=n_{1}, n_{2}<n / 2
$$

2. For each scale/angle pair $(j, l)$, interpolate $f\left[n_{1}, n_{2}\right]$ to obtain sampled values $f\left[n_{1}, n_{2}-\right.$ $\left.n_{1} \tan \theta_{l}\right]$ for $\left(n_{1}, n_{2}\right) \in P_{j}$.

3. Multiply the interpolated object $\mathrm{f}$ with the parabolic window $U_{j}$, effectively localizing f near the parallelogram with orientation $\theta_{l}$, and obtain

$$
f\left[n_{1}, n_{2}\right]=f\left[n_{1}, n_{2}-n_{1} \tan \theta_{l}\right] U_{j}\left[n_{1}, n_{2}\right]
$$

4. Apply inverse 2D FFT to each $f_{j, l}$, hence collecting the discrete coefficients $c^{D}(j, l, k)$

\subsection{Contourlet Transform}

Contourlet transform is a form of directional multiresolution image representation and made up of a smooth region's partition by smooth boundaries. Contourlet transform is implemented based on 
two types are Laplace pyramid and directional filter bank. The contourlet transform is based on basis functions with flexible aspect ratios and different directions in multiple scales. It has a small redundancy unlike other transforms. The extension of the curvelet transform is used to capture curves instead of points, but it provides for directionality. Contourlet has offered a high degree of directionality and anisotropy and not only access the main features of wavelets like multiscale and time frequency localization. The consulate is achieving critical sampling, but it takes different and flexible number of directions at each scale [13].

\section{Contourlet Transform algorithm}

Step 1: The input image has four frequency components like LL (Low Low), LH (Low High), HL (High Low) and HH (High High).

Step 2: At each stage, Laplacian pyramid produces low pass output (LL) and a band pass output (LH, HL, HH).

Step3: The band pass output is passed into a directional filter bank, which produces the results as contourlet coefficient. Then the low pass output is again passed through the laplacian pyramid to produce more coefficients. The process is repeated until the fine details of the image are retrieved.

Step 4: Finally, the image is reconstructed by applying the inverse contourlet transform.

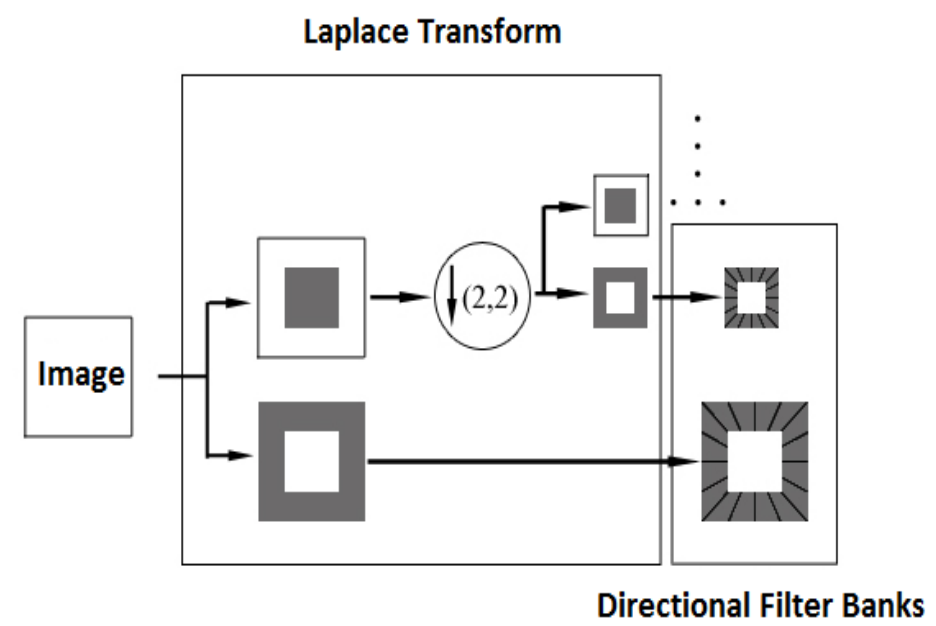

Fig. 3 Decompostion of contourlet transform

\subsection{Local ternary pattern}

Local ternary pattern (LTP) is a texture operator has higher dimensionality and more discriminating than LBP. LBP has threshold pixels into 0 and 1 value, but LTP uses a threshold pixel in 0,1 and -1 values. Considering $\mathrm{p}$ is a neighboring pixel, $\mathrm{c}$ as the center pixel and $\mathrm{k}$ as the threshold constant [14], [15].

$$
\mathrm{F}(\mathrm{x})= \begin{cases}1, & \text { if } \mathrm{p}>\mathrm{c}+\mathrm{k} \\ 0, & \text { if } \mathrm{p}>\mathrm{c}-\mathrm{k} \text { and } \mathrm{p}<\mathrm{c}+\mathrm{k} \\ -1, & \text { if } \mathrm{p}<\mathrm{c}-\mathrm{k}\end{cases}
$$




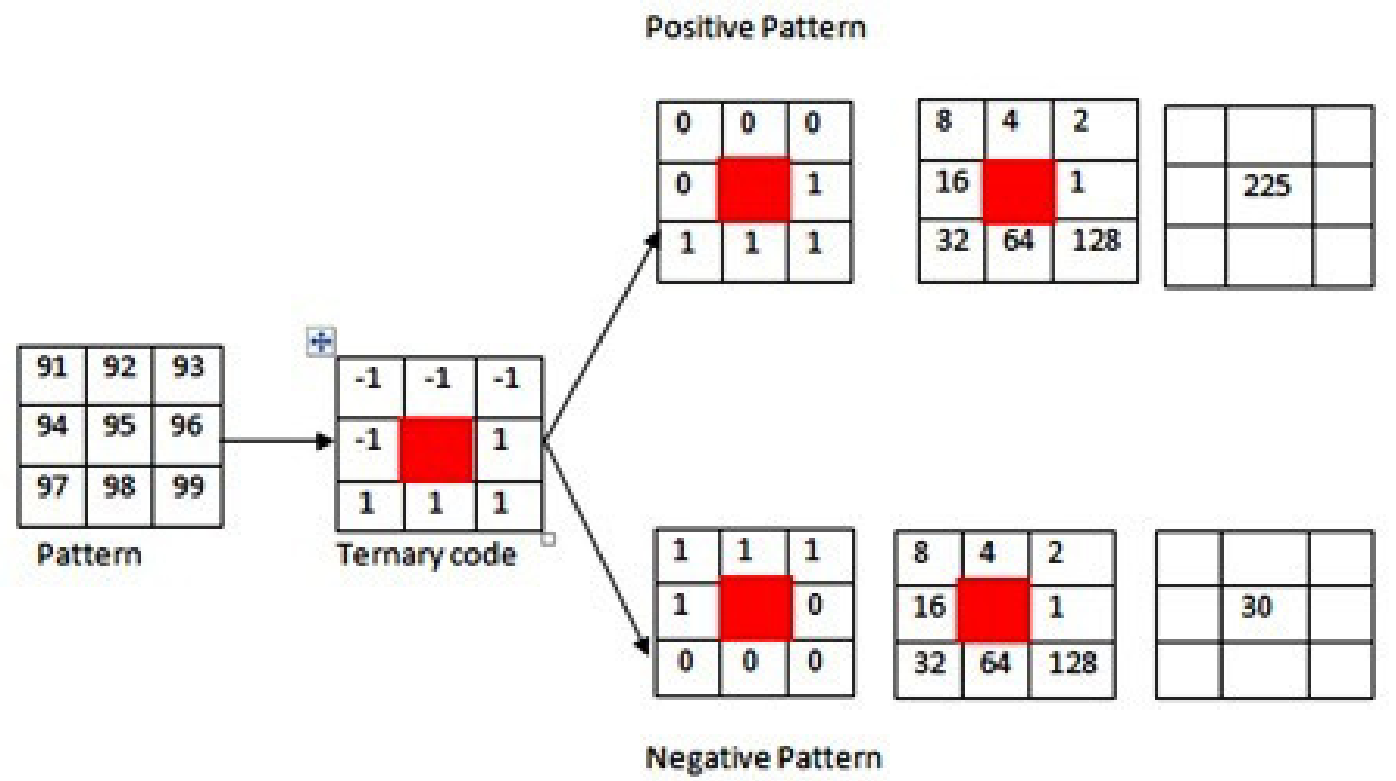

Fig. 4 Calculation of LTP

\section{LTP algorithm}

1 Consider a pixel surrounded by eight neighbors.

2 Every pixel in a cell, left- top, left-middle, left-bottom, right-top, etc. are compared in the center pixel to each of its 8 neighbors.

3 The normal pixel values in an image form a value in the pattern matrix.

4 To extract the LTP for the center pixel, C, Where the center pixel's value is greater than the neighbor's value, assigns "1", center pixel value is lesser than neighbors value, assign"- 1 " and center pixel value is equal to neighbors value, assign " 0 ".

5 Split as positive pattern and negative pattern.

6 Finally, the local ternary pattern value is calculated.

\section{Classification}

Classification is the process in which ideas and objects are recognized, differentiated, and understood. The Deep neural network (DNN)) is the classification techniques used for MRI brain tumor images.

\subsection{Deep Neural network}

Deep neural network (DNN) is a multilayer neural network model that has more than one layer of hidden units between its inputs and its outputs. The two important processes are used in the classification are training and testing phase. In the training phase, the features of training data are trained using deep learning classifier. Commonly used neural network uses back propagation algorithm. But it is not adequate for training neural networks with many hidden layers on large amounts of data. Deep Neural Networks that contain many layers of nonlinear hidden units and a very large output layer. Deep neural networks have deep architectures which have the capacity to learn more complex models than shallow ones [16] - [18]. 


\section{DNN algorithm}

1) In the first phase, greedily train subsets of the parameters of the network using a layerwise and supervised learning criterion, by repeating the following steps for each layer $(\mathrm{i} \in\{1, \ldots, 1\})$

Until a stopping criteria is met, iterate through the training database by

(a) mapping input training sample $\mathrm{x}_{t}$ to representation $h^{i-1}\left(\mathrm{x}_{t}\right)$ (if $i>1$ ) and hidden representation $\mathrm{h}^{\mathrm{i}}\left(\mathrm{x}_{\mathrm{t}}\right)$

(b) updating parameters $b^{i-1}$, bi and $W^{i}$ of layer $i$ using some supervised learning algorithm. also, initialize (e.g., randomly) the output layer parameters $\mathrm{b}^{1+1}, \mathrm{~W}^{1+1}$

2) In the second and final phase, fine-tune all the parameters $\theta$ of the network using backpropagation and gradient descent on a global supervised cost function $C\left(\mathrm{x}_{\mathrm{t}}, \mathrm{y}_{\mathrm{t}}, \theta\right)$ with input $\mathrm{x}_{\mathrm{t}}$ and label $\mathrm{y}_{\mathrm{t}}$, that is, trying to make steps in the direction $E\left[\frac{\partial c\left(\alpha_{\mathrm{t}} y_{\mathrm{t}} \theta\right)}{\partial \theta}\right]$

\section{Performance Metrics}

Sensitivity, specificity, accuracy, error rate and f-measure are used to measure the performance of curvelet transform, contourlet transform and local ternary pattern.

\subsection{Sensitivity}

Specificity measures the proportion of positives which are correctly identified, such as the percentage of sick people who are correctly identified as not having the condition, sometimes called the true negative rate [19].

$$
\text { Sensitivity }=\frac{T P}{T P+F N}
$$

Where, TP - True Positive (equivalent with hit)

FN - False Negative (equivalent with miss)

TN - True Negative (equivalent with correct rejection)

\subsection{Specificity}

Specificity measures the proportion of negatives which are correctly identified, such as the percentage of healthy people who are correctly identified as not having the condition, sometimes called the true negative rate [19].

$$
\text { Specificity }=\frac{T N}{(F F+T N)}
$$

Where, TN - True Negative (equivalent with correct rejection)

FN - False Negative (equivalent with miss)

TN - True Negative (equivalent with correct rejection) 
International Journal in Foundations of Computer Science \& Technology (IJFCST) Vol.5, No.6, November 2015

\subsection{Accuracy}

Accuracy is the measurement system, which measure the degree of closeness of measurement between the original value and the extracted value [19].

$$
\text { Accuracy }=\frac{T P+T N}{(T P+F P+T N+F N)} \times 100
$$

Where TP - True Positive (equivalent with hit)

FN - False Negative (equivalent with miss)

$\mathrm{TN}$ - True Negative (equivalent with correct rejection)

\subsection{Error Rate}

Error rate is used to compare an estimate value to an exact value [19].

Error rate $=100-\mathrm{A}$

\section{A- Accuracy}

\subsection{F- measure}

$\mathrm{F}$ - measure is a harmonic mean of precision and recall [19].

$$
\text { F- measure }=\frac{2 \tau p}{(2 T P+F P+F N)}
$$

Where TP- number of true positive

FP- number of false positive

FN- number of false negative

Table. 1 Computing Sensitivity for DNN in Curvelet transform, Contourlet transform and Local ternary pattern

\begin{tabular}{|c|c|c|c|}
\hline Sensitivity (\%) & Img 1 & Img 712 & Img 978 \\
\hline Curvelet Transform & 52 & 52 & 52 \\
\hline Contourlet Transform & 52 & 52 & 52 \\
\hline Local Ternary Pattern & 57 & 60 & 57 \\
\hline
\end{tabular}


International Journal in Foundations of Computer Science \& Technology (IJFCST) Vol.5, No.6, November 2015

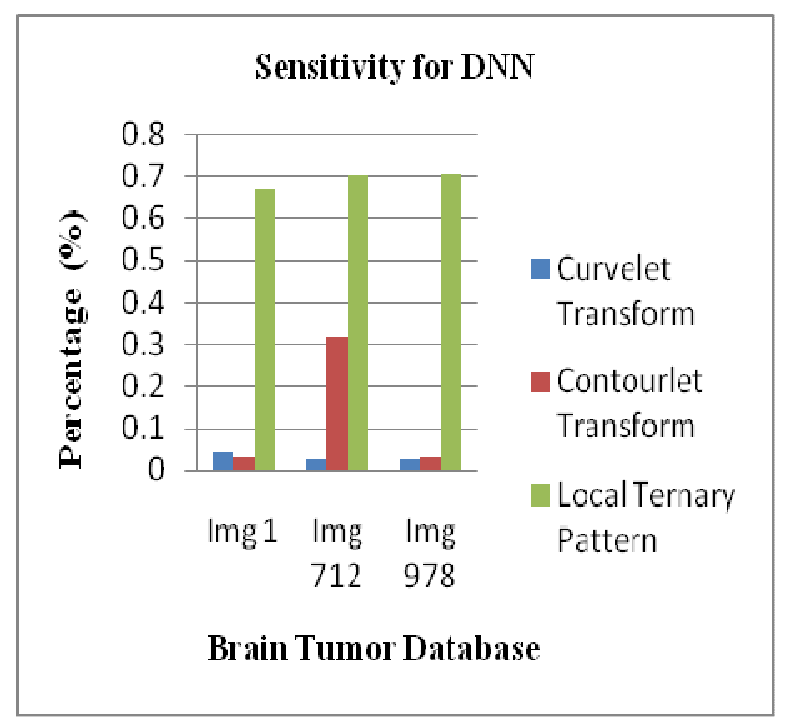

Fig. 5 Retrieval performance of Sensitivity for DNN in Curvelet transform, Contourlet transform and Local ternary pattern

Table. 2 Computing Specificity for DNN in Curvelet transform, Contourlet transform and Local ternary pattern

\begin{tabular}{|c|c|c|c|}
\hline Specificity (\%) & Img 1 & Img 712 & Img 978 \\
\hline Curvelet Transform & 38 & 38 & 38 \\
\hline Contourlet Transform & 38 & 38 & 38 \\
\hline Local Ternary Pattern & 51 & 51 & 51 \\
\hline
\end{tabular}

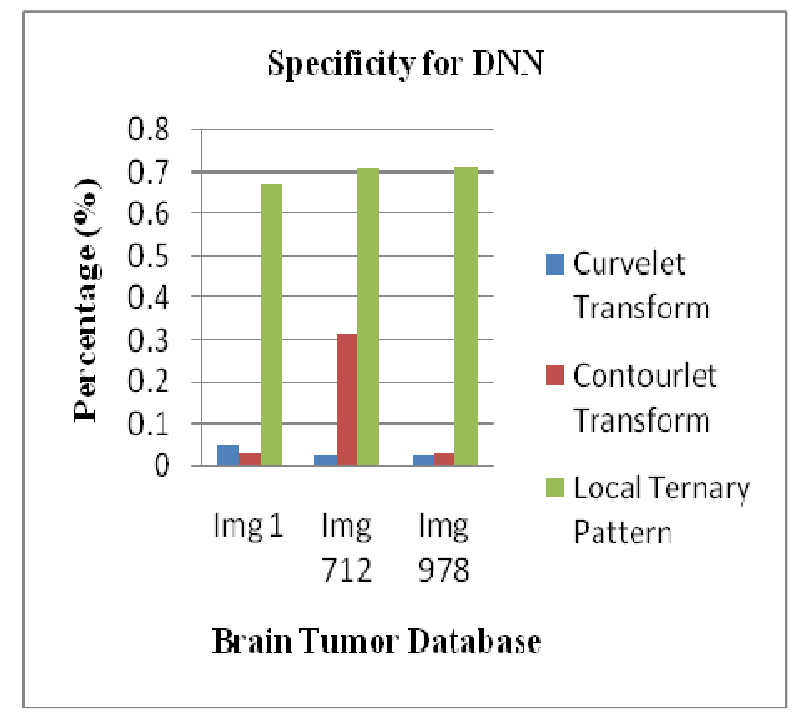

Fig. 6 Retrieval performance Specificity for DNN in Curvelet transform, Contourlet transform and Local ternary pattern 
International Journal in Foundations of Computer Science \& Technology (IJFCST) Vol.5, No.6, November 2015

Table. 3 Computing Accuracy for DNN in Curvelet transform, Contourlet transform and Local ternary pattern

\begin{tabular}{|c|c|c|c|}
\hline Accuracy (\%) & Img 1 & Img 712 & Img 978 \\
\hline Curvelet Transform & 97.5 & 97.5 & 97.5 \\
\hline Contourlet Transform & 97.5 & 97.5 & 97.5 \\
\hline Local Ternary Pattern & 20 & 15 & 20 \\
\hline
\end{tabular}

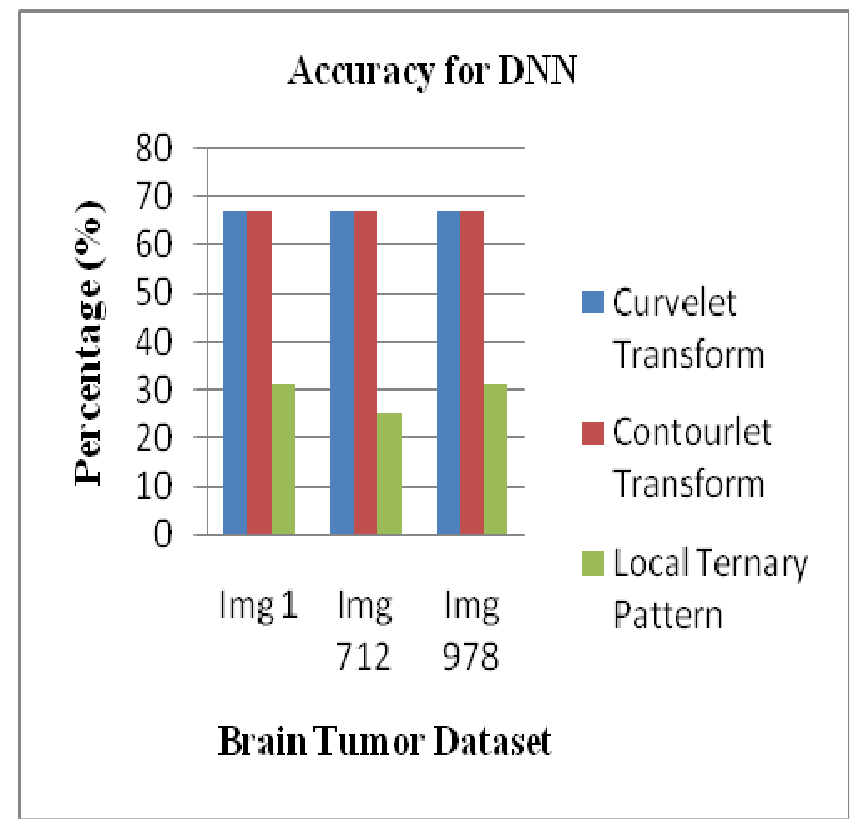

Fig. 7 Retrieval performance of Accuracy for DNN in Curvelet transform, Contourlet transform and Local ternary pattern

Table. 4 Computing Error rate for DNN in Curvelet transform, Contourlet transform and Local ternary pattern

\begin{tabular}{|c|c|c|c|}
\hline Error rate (\%) & Img 1 & Img 712 & Img 978 \\
\hline Curvelet Transform & 2.5 & 2.5 & 2.5 \\
\hline Contourlet Transform & 2.5 & 2.5 & 2.5 \\
\hline Local Ternary Pattern & 80 & 85 & 80 \\
\hline
\end{tabular}


International Journal in Foundations of Computer Science \& Technology (IJFCST) Vol.5, No.6, November 2015

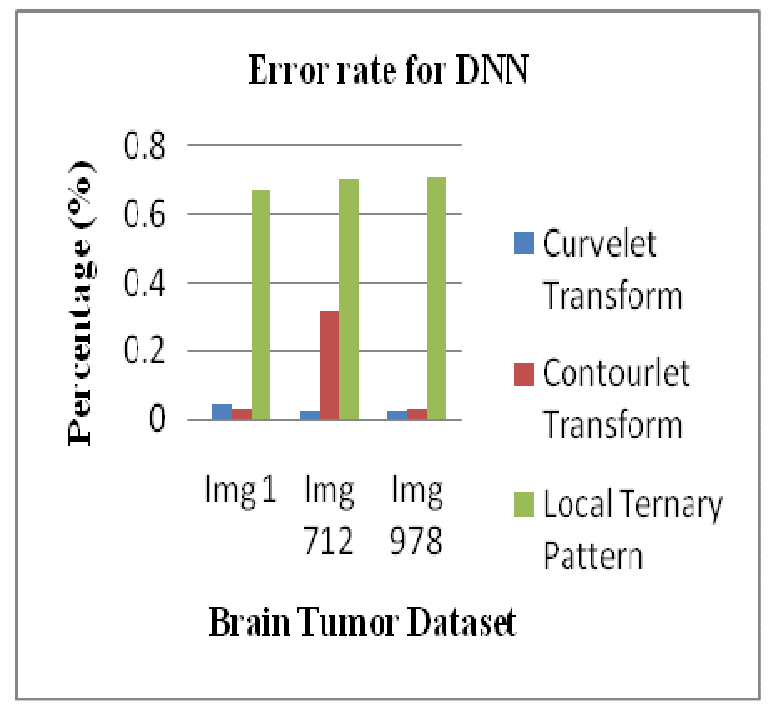

Fig. 8 Retrieval performance of Error rate for DNN in Curvelet transform, Contourlet transform and Local ternary pattern

Table. 5 Computing F-measure for DNN in Curvelet transform, Contourlet transform and Local ternary pattern

\begin{tabular}{|c|c|c|c|}
\hline F-measure (\%) & Img 1 & Img 712 & Img 978 \\
\hline Curvelet Transform & 67 & 67 & 67 \\
\hline Contourlet Transform & 67 & 67 & 67 \\
\hline Local Ternary Pattern & 31 & 25 & 31 \\
\hline
\end{tabular}

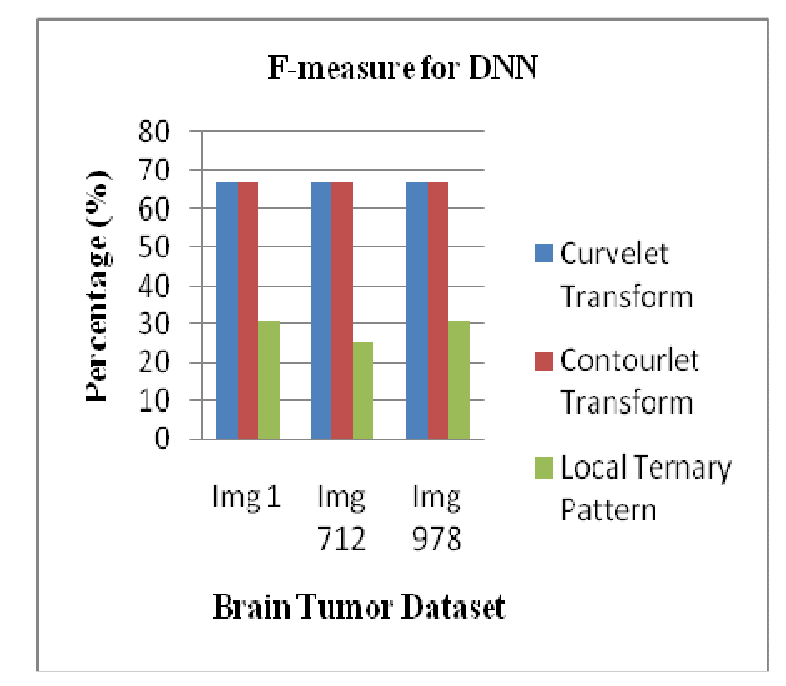

Fig. 9 Retrieval performance of F-measure for DNN in Curvelet transform, Contourlet transform and Local ternary pattern 
International Journal in Foundations of Computer Science \& Technology (IJFCST) Vol.5, No.6, November 2015

Table. 6 Computing total time taken for DNN in Curvelet transform, Contourlet transform and Local ternary pattern

\begin{tabular}{|c|c|c|c|}
\hline Total time taken $(\mathrm{Sec})$ & Img 1 & Img 712 & Img 978 \\
\hline Curvelet Transform & 0.2819 & 0.0871 & 0.0922 \\
\hline Contourlet Transform & 0.0961 & 0.0843 & 0.0847 \\
\hline Local Ternary Pattern & 0.0964 & 0.0929 & 0.0931 \\
\hline
\end{tabular}

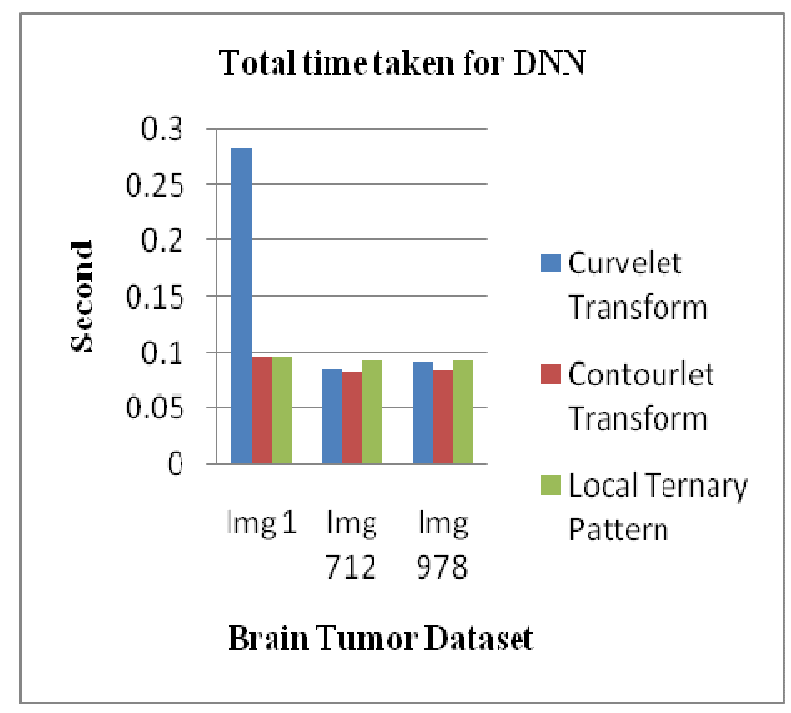

Fig. 10 Retrieval performance of total time taken for DNN in Curvelet transform, Contourlet transform and Local ternary pattern

\section{EXPERIMENTAL RESULTS}

To evaluate the overall performance, the brain tumor image are the type of both sagittal and axial. The MRI brain tumor image database contains 1000 images. Brain images contain 5 classes and each class has 200 images. The resolution of the image is $256 \times 256$. The performance of the method can be evaluated by using sensitivity, specificity, accuracy, error rate and f-measure. For evaluation, DNN classification accuracy is as follows: curvelet transform- 97.5\%, contourlet transform- $97.5 \%$ and local ternary pattern- $18.33 \%$. The average time taken is as follows: Curvelet transform- $0.155 \mathrm{sec}$, contourlet transform- $0.088 \mathrm{sec}$ and local ternary pattern- 0.094 sec. The sensitivity, specificity, error rate and f-measure values are evaluated.

The Experiment result shows that the texture feature techniques contourlet transform is better than curvelet transform and local ternary pattern. In terms of time values, Contourlet transform is better than curvelet transform and local ternary pattern. So, contourlet transform achieves better performance. 


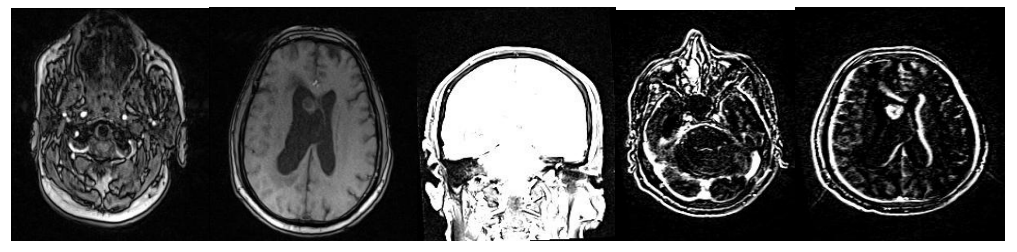

Fig. 11 Sample images

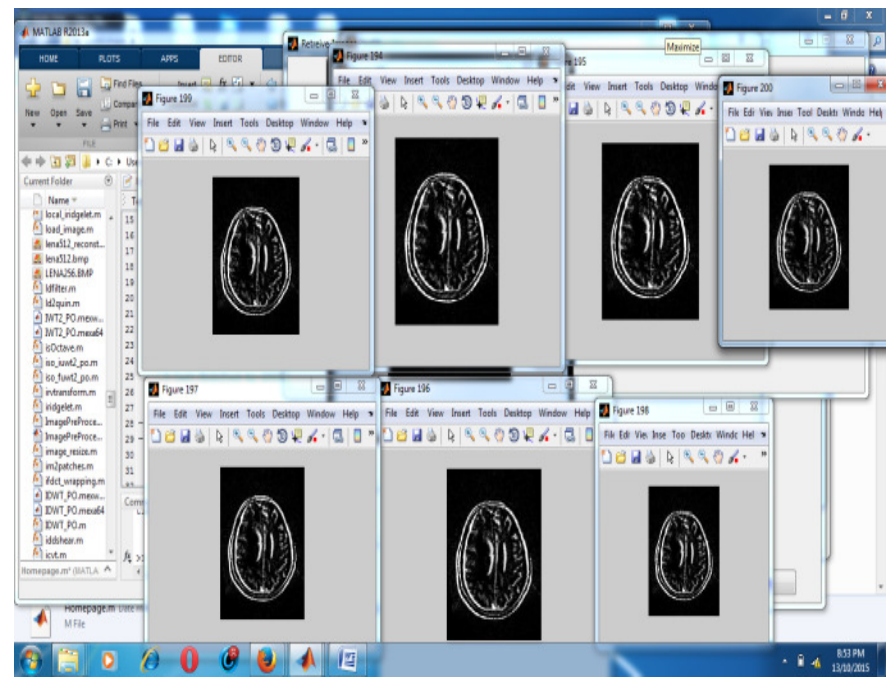

Fig. 12 Retrieval of brain tumor images

\section{Conclusion}

In this paper, the performance of texture feature extraction for MRI brain tumor image retrieval is evaluated. This feature extraction technique is used for medical image diagnosis in the clinical domain. The performance of the method has two different steps are feature extraction process and classification. The Curvelet transform, contourlet transform and local ternary pattern techniques are used for texture feature extraction. To classify the brain tumor image for supervised learning algorithm namely DNN is used. It is inferred from the results that contourlet transform using DNN classifier outperform other techniques like curvelet transform and local ternary pattern. Contourlet transform achieves better performance. This method is efficient for classification of the human brain tumor database and similar image. It increases the retrieval time and improves the retrieval accuracy significantly.

\section{REFERENCES}

[1] Rui, Yong, Thomas S. Huang, and Shih-Fu Chang. "Image retrieval: Current techniques, promising directions, and open issues." Journal of visual communication and image representation 10, no. 1 (1999): 39-62.

[2] Somasundaram. K., and T. Kalaiselvi. "Automatic brain extraction methods for T1 magnetic resonance images using region labeling and morphological operations." Computers in Biology and Medicine 41.8 (2011): 716-725.

[3] Wells III, Williams M., W. Eric L. Grimson, Ron Kikinis, and Ferenc A. Jolesz. "Adaptive segmentation of MRI data." Medical Imaging, IEEE Transactions on 15, no. 4 (1996): 429-442. 
[4] Unay, Devrim, Ahmet Ekin, and Radu S. Jasinschi. "Local structure-based region-of-interest retrieval of brain MR images." Information Technology in Biomedicine, IEEE Transactions on 14.4 (2010): 897-903.

[5] Shen, Tian, Hongsheng Li, and Xiaolei Huang. "Active volume models for medical image segmentation." Medical Imaging, IEEE Transactions on 30.3 (2011): 774-791.

[6] Selvathi, D., R. S. Ram Prakash, and S. Thamarai Selvi. "Performance evaluation of kernel based techniques for brain MRI data classification." Conference on Computational Intelligence and Multimedia Applications, 2007. International Conference on. Vol. 2. IEEE, 2007.

[7] Cai, Weidong, Sidong Liu, Lingfeng Wen, Stefan Eberl, Michael J. Fulham, and Dagan Feng. "3D neurological image retrieval with localized pathology-centric CMRGlc patterns." In Image Processing (ICIP), 2010 17th IEEE International Conference on, pp. 3201-3204. IEEE, 2010.

[8] Li, Jin, Hong Liang, and Hong Yu. "Brain pathological image retrieval based on correlation of visual feature and report-assisted text." Mechatronics and Automation, 2008. ICMA 2008. IEEE International Conference on. IEEE, 2008.

[9] Li, Weijuan, et al. "Meticulous classification using support vector machine for brain image retrieval." Medical Image Analysis and Clinical Applications (MIACA), 2010 International Conference on. IEEE, 2010.

[10] Castelli, Vittorio, and Lawrence D. Bergman, Eds. Image databases: search and retrieval of digital imagery. John Wiley \& Sons, 2004.

[11] Sumana, Ishrat Jahan, Md Monirul Islam, Dengsheng Zhang, and Guojun Lu. "Content based image retrieval using curvelet transform." In Multimedia Signal Processing, 2008 IEEE 10th Workshop on, pp. 11-16. IEEE, 2008.

[12] Prabha .S and Sasikala .M,"Texture Classification Using Curvelet Transform” International Journal of Advancements in Research \& Technology, Volume 2, Issue4, April-2013 249 ISSN 2278-7763.

[13] Manju, K., and Smita Tikar. "Contourlet Transform and PNN Based Brain Tumor Classification." International Journal of Innovative Research and Development (2014).

[14] Murala, Subrahmanyam, R. P. Maheshwari, and R. Balasubramanian. "Local tetra patterns: a new feature descriptor for content-based image retrieval." Image Processing, IEEE Transactions on 21, no. 5 (2012): 2874-2886.

[15] https://en.wikipedia.org/wiki/Local_ternary_patterns

[16] Larochelle, Hugo, Yoshua Bengio, Jérôme Louradour, and Pascal Lamblin. "Exploring strategies for training deep neural networks." The Journal of Machine Learning Research 10: 1-40, September 2009.

[17] Hinton, Geoffrey, Li Deng, Dong Yu, George E. Dahl, Abdel-rahman Mohamed, Navdeep Jaitly, Andrew Senior et al. "Deep neural networks for acoustic modeling in speech recognition: The shared views of four research groups." Signal Processing Magazine, IEEE 29, no. 6: 82-97, November 2012.

[18] Gladis Pushpa V.P, Rathi and Palani .S, "Brain Tumor Detection and Classification Using Deep Learning Classifier on MRI Images" Research Journal of Applied Sciences Engineering and Technology10(2): 177-187, May-2015, ISSN:20407459.

[19] https://en.wikipedia.org/wiki/Sensitivity and specificity 